\title{
The cytoplasmic droplet may be indicative of sperm motility and normal spermiogenesis
}

\begin{abstract}
Hui Xu ${ }^{1, *}$, Shui-Qiao Yuan ${ }^{1,2, *}$, Zhi-Hong Zheng ${ }^{1}$ and Wei Yan ${ }^{1,2}$
Although the cytoplasm of spermatids is removed at the end of spermiogenesis, a tiny portion is usually retained in the sperm flagellum, which is termed the cytoplasmic droplet (CD) in mammals. CDs are believed to play a role in sperm volume adaptation. However, we have noticed that epididymal spermatozoa that display initial (flagellation in situ) and progressive motility mostly possess CDs, whereas spermatozoa without CDs are rarely motile, suggesting that CDs have a role in motility development during sperm epididymal maturation. In the present study, we analyzed the relationship between the presence or absence of CDs, motility development and positional changes of CDs during sperm epididymal maturation in mice and monkeys. We also examined CDs on spermatozoa of three knockout mouse lines with late spermiogenic defects. Our data suggest that the $C D$ is a normal organelle transiently present exclusively on epididymal spermatozoa, and normal $C D$ morphology and location are associated with normal motility development during epididymal maturation of spermatozoa. Abnormal CD formation, e.g., a complete lack of CDs or ectopic CDs, is indicative of defective spermiogenesis. If CDs are essential for sperm motility development, then CDs may represent an ideal drug target for the development of non-hormonal male contraceptives.
\end{abstract}

Asian Journal of Andrology (2013) 15, 799-805; doi:10.1038/aja.2013.69; published online 17 June 2013

Keywords: cytoplasmic droplets; epididymis; fertility; mouse; semen analysis; spermiogenesis; sperm motility

\section{INTRODUCTION}

In all mammalian species, spermatozoa are released from the seminiferous epithelium through a process termed spermiation, during which the cytoplasm of elongated spermatids is removed and the ultimate form of male gametes, spermatozoa, are released to the lumen of the seminiferous tubules. ${ }^{1}$ Epididymal spermatozoa possess a droplet-like structure usually located at the middle piece or midprincipal piece junction of the sperm flagellum. ${ }^{2-6}$ Retzius first described this structure in 1909 and termed it cytoplasmic droplet (CD). ${ }^{7}$ In mice, CDs appear to be derived from the spermatid cytoplasm on the basis of their ultrastructure. ${ }^{2,8,9} \mathrm{CDs}$ are believed to be involved in osmolality regulation because when spermatozoa are challenged with low osmolality, the flagellum of epididymal spermatozoa becomes coiled at the position of the CD. ${ }^{10-12}$ Mice with defects in several water channel proteins tend to display coiled sperm flagella at the position of $\mathrm{CD}$ attachment. ${ }^{10,13}$ However, it remains unknown why ejaculated murine sperm, most of which do not have CDs, rarely display flagellum coiling when they are deposited into the female reproductive tract where the osmolality is generally lower than that of semen or the cauda epididymidis (e.g. $\sim 300$ mosmol $^{-1}$ in the uterine cavity vs. $\sim 440$ mosmol $\mathrm{l}^{-1}$ in the cauda epididymidis). ${ }^{10}$

The confusion in CD morphology and location between mouse and human spermatozoa may result from the fact that mouse epididymal spermatozoa are usually used for studies, whereas human ejaculated, rather than epididymal, spermatozoa are analyzed. A morphological study on human ejaculated spermatozoa has shown that there are two types of cytoplasm on human sperm cells: one representing remnant cytoplasm usually attached to any portion of the sperm flagellum, and the 'true' CDs representing that more stably attached to the neck region, which can easily be lost or become invisible owing to solvent dehydration in staining preparations. ${ }^{14-16}$ However, in mouse epididymal spermatozoa, CDs are clearly visible in the phase contrast microscope and mostly located to the middle piece of the flagella. ${ }^{17}$ The difference in morphology and position between human and mouse sperm CDs may reflect species differences. Despite the claim that CDs function as an osmolality regulatory apparatus, controversy remains because in human spermatozoa, low osmolality results in curled flagellar tips instead of bending at the neck. ${ }^{11,12,18}$ Nevertheless, the function of CDs beyond osmolality regulation has been scarcely explored.

In mice, about $30 \%-40 \%$ of cauda epididymidal spermatozoa collected into human tubal fluid (HTF) medium never become motile. ${ }^{19}$ Similarly, about $20 \%-40 \%$ of human spermatozoa in ejaculates are immotile, ${ }^{20}$ suggesting that a large proportion of spermatozoa produced are defective, at least in their ability to develop motility. We have long noticed that the majority of mouse epididymal spermatozoa that do not become motile upon collection in HTF medium have no CDs, suggesting a positive correlation between the presence of CDs and the ability to express motility. We have also observed that disruption of spermiogenesis due to gene inactivation results in an abnormal

${ }^{1}$ Department of Laboratory Animal Medicine, China Medical University, Shenyang 110001, China and ${ }^{2}$ Department of Physiology and Cell Biology, University of Nevada School of Medicine, Reno, NV 89557, USA

* These authors contributed equally to this work.

Correspondence: Professor W Yan (wyan@medicine.nevada.edu)

Received: 6 March 2013; Revised: 4 April 2013; Accepted: 30 April 2013; Published online: 17 June 2013 
location of CDs or an increased proportion of spermatozoa without $\mathrm{CDs},{ }^{21-23}$ suggesting that normal CDs may be a hallmark of normal spermiogenesis. In the present study, we systematically studied the correlation between the presence or absence of CDs and sperm motility in three commonly used laboratory mouse strains and cynomolgus monkeys. We also analyzed CDs in three knockout (KO) mouse lines with late spermiogenic disruptions. Our data suggest that CDs are not only a good predictor of sperm motility potential but also a hallmark of normal spermiogenesis.

\section{MATERIALS AND METHODS}

\section{Experimental animals}

Three mouse strains (C57BL/6J, ICR and FVB) were maintained in a temperature- and humidity-controlled animal facility with free access to water and food at the Laboratory Animal Center in China Medical University. Adult male mice ( $>8-16$ weeks of age) were used as source of epididymal spermatozoa. Three KO mouse lines $\left(\operatorname{Tnp} 1^{-/-}\right.$, Tnp $1^{-/-}-\mathrm{Hisl}^{-/-}$and Spem $1^{-/-}$) were housed in a temperatureand humidity-controlled animal facility in the University of Nevada, Reno, NV, USA, with free access to water and food. Male mice of 8-12 weeks of age were used as source of epididymal spermatozoa. The animal protocols were approved by the Institutional Animal Care and Use Committee of the University of Nevada (Reno, NV, USA).

Cynomolgus monkey (Macaca fascicularis) epididymides were obtained from Charles River Preclinical Services (Sparks, NV, USA). The protocol for euthanizing monkeys was approved by the Institutional Animal Care and Use Committee which assures compliance with the United States Department of Agriculture, Public Health Service Office of Laboratory Animal Welfare Policy and the Animal Welfare Act (Charles River Laboratories, Preclinical Services, Sparks, $\mathrm{NV}$, USA). Adult male monkeys were sedated with Ketamine (10 mg $\mathrm{kg}^{-1}$ body weight) via intramuscular injection into the quadriceps. They were then given Beuthanasia-D solution containing pentobarbital sodium and phenytoin sodium (200 $\mathrm{mg} \mathrm{kg}^{-1}$ body weight) via intravenous injection followed by exsanguination. The epididymidis were dissected, placed in cold KRBS and transported to the laboratory within $30 \mathrm{~min}$ of dissection.

Data on normal CD positional changes and correlations with motility development were collected from 10 adult wild-type mice for each of the three strains analyzed and 10 adult monkeys. A total of five adult KO mice with each of the four genotypes (wild-type, $\operatorname{Tn} p 1^{-1-}, \operatorname{Tnp1}^{-1-}$ $\mathrm{Hils}^{-/-}$and Spem1 ${ }^{-/-}$) were used to collect data on the presence or absence of CDs and ectopic CDs on epididymal spermatozoa.

\section{Preparation of murine epididymal spermatozoa}

Mouse epididymides were dissected into small pieces $(\sim 5 \mathrm{~mm} \times 5 \mathrm{~mm})$ in a $1.5 \mathrm{ml}$ Eppendorf tube containing $1 \mathrm{ml}$ of pre-warmed $\left(37^{\circ} \mathrm{C}\right)$ HTF-HEPES medium ( $\mathrm{pH} \mathrm{7.4,} 300 \mathrm{mosmol}^{-1}$; Irvine Scientific, Santa Ana, CA, USA) followed by incubation at $37{ }^{\circ} \mathrm{C}$ for $30 \mathrm{~min}$. Monkey epididymides were cut into small pieces $(\sim 5 \mathrm{~mm} \times 5 \mathrm{~mm})$ and incubated in $5 \mathrm{ml} \mathrm{HTF-HEPES}$ medium at $37{ }^{\circ} \mathrm{C}$ for $30 \mathrm{~min}$.

\section{CD identification and counting}

Suspensions of epididymidal spermatozoa were diluted to a concentration of $\sim 1$ million cells $\mathrm{ml}^{-1}$ and a small aliquot $(\sim 5 \mu \mathrm{l})$ was loaded onto a chamber slide of $20 \mu \mathrm{m}$ depth (Conception Technologies, San Diego, CA, USA). Movie clips of different fields were recorded by using an inverted phase contrast microscope equipped with a video camera (AxioVision; Carl Zeiss, Oberkochen, Germany). The number of spermatozoa with or without CDs was assessed at a later time. CDs were judged on their morphology and transillumination absorbance.

\section{Collection of ejaculated spermatozoa from the uterus}

CD1 females at the estrous phase were mated with stud C57BL/6J, ICR or FVB males. Vaginal plugs were checked every $30 \mathrm{~min}$, and once the vaginal plug was identified, the female CD1 mouse was immediately sacrificed, and the vagina and uterus were dissected into small pieces $(\sim 5 \mathrm{~mm} \times 5 \mathrm{~mm})$ in $2 \mathrm{ml}$ of HTF-HEPES medium followed by incubation at $37{ }^{\circ} \mathrm{C}$ for $15 \mathrm{~min}$. An aliquot of suspension $(\sim 5 \mu \mathrm{l})$ was transferred to a chamber slide of $20 \mu \mathrm{m}$ depth (Conception Technologies) for observation and movie recording. About 1000 spermatozoa were assessed at $\times 200$ magnification and the number with or without CDs was recorded.

\section{Statistical analyses}

Data are presented as mean \pm s.e.m., and statistical differences between datasets were assessed by one-way ANOVA by using SPSS16.0 software. Differences were considered significant at the level of $P<0.05$.

\section{RESULTS}

CDs are mainly located on the middle piece of epididymal sperm flagella

Although CDs could be found in any position along the flagella of epididymal spermatozoa in mice (Figure 1A), the majority $(80 \%-$ 97\%) of CDs were found on the middle piece of sperm flagella in all three strains of mice analyzed (Figure 2). In the corpus epididymidis, spermatozoa with CDs on the neck region and the principal or end piece accounted for $6 \%-19 \%$ and $0 \%-3 \%$, respectively (Figure 2), whereas in the cauda epididymidis, proportions of spermatozoa with CDs on the necks were lower $(1 \%-2 \%)$, and those with CDs on the principal or end piece remained low $(2 \%-4 \%)$ in all three strained examined (Figure 2). Thus, it appeared that with spermatozoa transiting from the caput to the cauda epididymidis, CDs of a small proportion of cells migrated from an upper (neck or mid-piece) to a lower (mid-principal junction, principal or end piece) position (Figure 2). Nevertheless, the vast majority of CDs (80\%-95\%) remained located at the mid-piece of sperm flagella throughout their journey through the epididymis in mice.

We also analyzed spermatozoa collected from the monkey epididymis. CDs were mainly found on the neck, middle piece, mid-principal junction and end piece of the epididymal spermatozoa in monkeys (Figure 1B). Interestingly, the majority of monkey caput epididymidal spermatozoa ( $\sim 92 \%$ ) displayed CDs on the neck, whereas the majority of CDs $(88 \%-89 \%)$ of corpus and cauda epididymidal spermatozoa were found on the mid-piece (Figure 2), demonstrating a descending movement of CDs as spermatozoa transited through the epididymis in monkeys. These data are consistent with previous reports, ${ }^{17,24}$ suggesting a distal migration of CDs from the neck region to mid or mid-principal piece junction in general during the sperm epididymal transition.

\section{Most epididymal spermatozoa that can develop motility possess cytoplasmic droplets}

Spermatozoa collected from the caput and corpus epididymidis did not have progressive motility. Instead they displayed initial motility characterized by non-progressive flagellation in situ in the HTF medium (Supplementary Movies 1 and 2). In contrast, cauda epididymidal spermatozoa displayed mostly progressive (i.e., forward) motility after incubation in the HTF medium (Supplementary Movie 3). In 
A
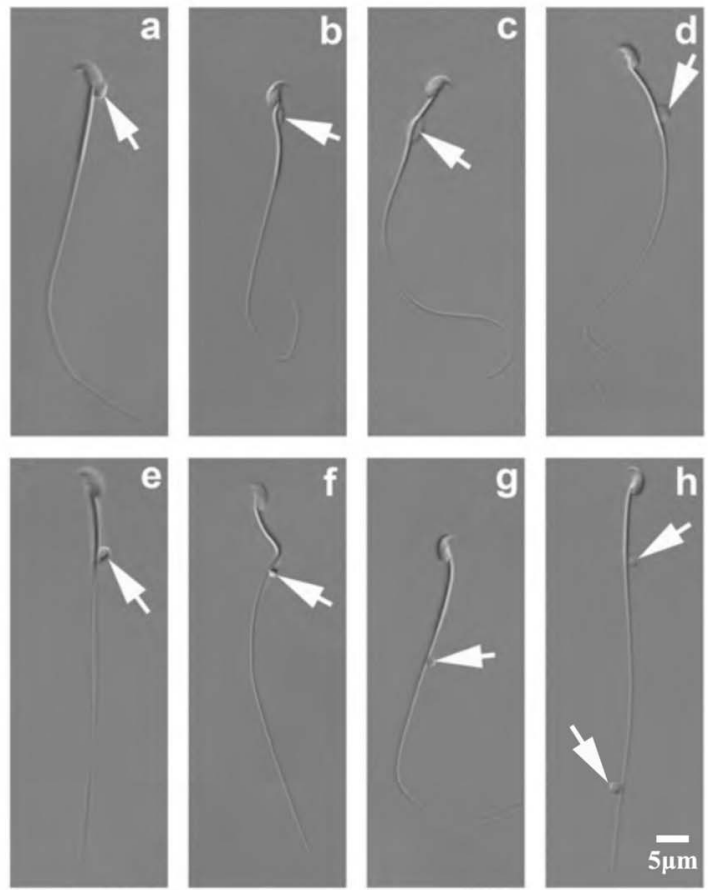

B
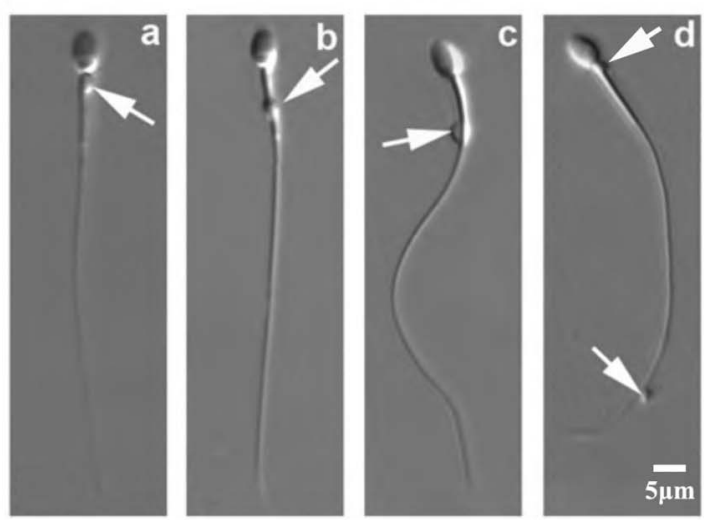

Figure 1 Positions of CDs on mouse and monkey epididymal spermatozoa. (A) DIC microscopic images showing various positions of CDs on mouse epididymal spermatozoa. Arrows point to CDs, which can be located at the head (a), the neck (b), the middle piece (c), the mid-principal piece junction (d-f), the principal piece $(\mathbf{g})$ and the end piece (h) of the flagellum. (B) DIC microscopic images showing various positions of CDs on monkey epididymal spermatozoa. Arrows point to $C D s$, which can be located at the neck (a), the middle piece $(\mathbf{b})$, the midprincipal piece junction (c), and the principal/end piece (d) (scale bar $=5 \mu \mathrm{m})$. $\mathrm{CD}$, cytoplasmic droplet; DIC, differential interference contrast.

this study, all flagellating spermatozoa were regarded as motile. Among the caput epididymidal spermatozoa, the percentage of motile spermatozoa with CDs $(75 \%-88 \%)$ was significantly higher than that of immotile spermatozoa with CDs (29\%-41\%) in all of the three mouse strains (C57BL/6J, ICR and FVB) (Figure 3a). Similar results were observed in corpus and cauda epididymidal spermatozoa in all three strains examined (Figure $\mathbf{3 b}$ and $\mathbf{c}$ ). Moreover, in the C57BL/6J and ICR mouse strains, the percentage of motile spermatozoa with CDs drastically decreased from $84 \%-88 \%$ to $41 \%-47 \%$ from the caput to cauda epididymidis, respectively (Figure 3d), suggesting that CDs have been shed in a subset of spermatozoa during the epididymal transition from the caput to the cauda epididymidis. Interestingly, in

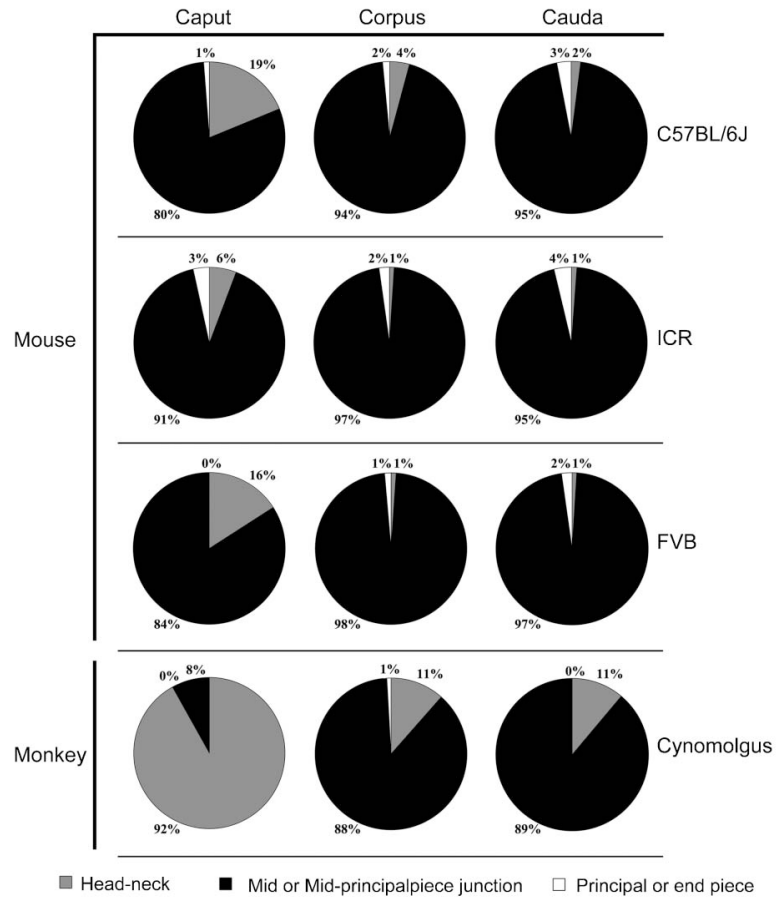

Figure 2 CD positional changes during epididymal transit in mice and monkeys. Pie charts showing proportions of the CD locations on spermatozoa collected from the caput, corpus and cauda epididymidis of mice (C57BL/6J, ICR and FVB strains) and monkeys. Data were collected from 10 mice for each of the three strains analyzed and from 10 monkeys. CD, cytoplasmic droplet.

the FVB strain, the percentage of motile spermatozoa with CDs displayed no significant changes in the three epididymal regions (Figure 3d), suggesting that CDs did not come off during the epididymal transition in this particular strain of mice.

We also analyzed the percentage of motile spermatozoa with or without CDs in three regions of the monkey epididymis (Figure 4). In monkeys, only $49 \%$ of caput epididymidal spermatozoa displayed initial motility, whereas the majority of corpus and cauda epididymidal sperm $(84 \%-86 \%)$ either displayed initial motility or developed progressive motility after incubation in HTF medium for $>30$ min (Figure 4a). Whereas when obtained from the caput epididymidis the majority of spermatozoa (91\%) possessed CDs, the number of CDbearing spermatozoa decreased to $\sim 50 \%$ when from the corpus epididymidis, and to only $\sim 16 \%$ when from the cauda epididymidis (Figure $4 \mathbf{b}$ ), suggesting that monkey spermatozoa gradually lose their CDs during their epididymal transition from the caput to cauda.

Spermatozoa without CDs or with ectopic CDs are common in mice with histologically indiscernible spermiogenic defects

Defective spermiogenesis refers to abnormal molecular or cellular events, which are usually caused by targeted gene mutation/deletion (e.g., gene KO), leading to the production of spermatozoa that are incompetent for fertilization or for supporting preimplantation development. ${ }^{23}$ Defective spermiogenesis may not necessarily display discernable disruptions in the seminiferous epithelium at histological levels (e.g. Spem1, CatSper3 and CatSper4 KO male mice). ${ }^{21,22}$ In the case of CatSper3 or CatSper4 KO spermatozoa, they look normal at both light and electron microscopic levels, but cannot fertilize eggs due to their inability to develop hyperactivated motility. ${ }^{22,23}$ Inactivation of genes exclusively expressed in late spermiogenesis tends to lead to 

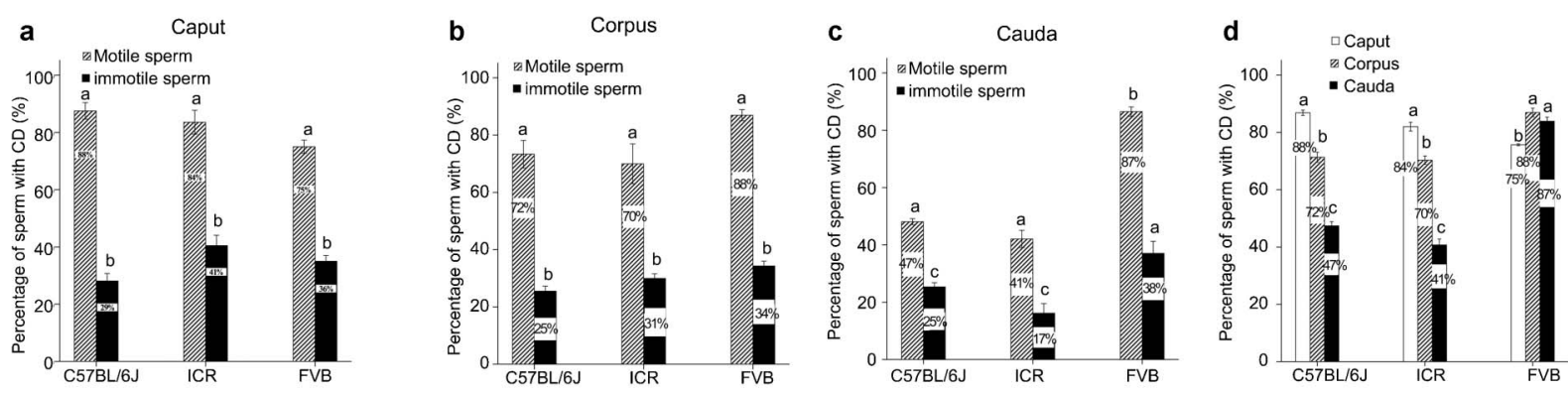

Figure 3 Presence of CDs is correlated with sperm motility. (a) Percentage of CD-bearing motile and immotile spermatozoa from the caput epididymidis of C57BL/6J, ICR and FVB mouse strains. (b) Percentage of CD-bearing motile and immotile spermatozoa from the corpus epididymidis of C57BL/6J, ICR and FVB mouse strains. (c) Percentage of CD-bearing motile and immotile spermatozoa from the cauda epididymidis of C57BL/6J, ICR and FVB mouse strains. (d) Percentage of motile CDbearing spermatozoa from the caput, corpus and cauda epididymidis of C57BL/6J, ICR and FVB mouse strains. Data are presented as mean \pm s.e.m. and datasets marked with different letters are statistically significant $(P<0.05, n=10)$. CD, cytoplasmic droplet.

defective spermiogenesis without discernable disruption to the seminiferous epithelial and sperm morphology. ${ }^{23}$

We have noticed that spermatozoa produced by $\mathrm{KO}$ mice with defective spermiogenesis tend to display aberrant motility characterized by reduced motility that quickly diminishes within a short period
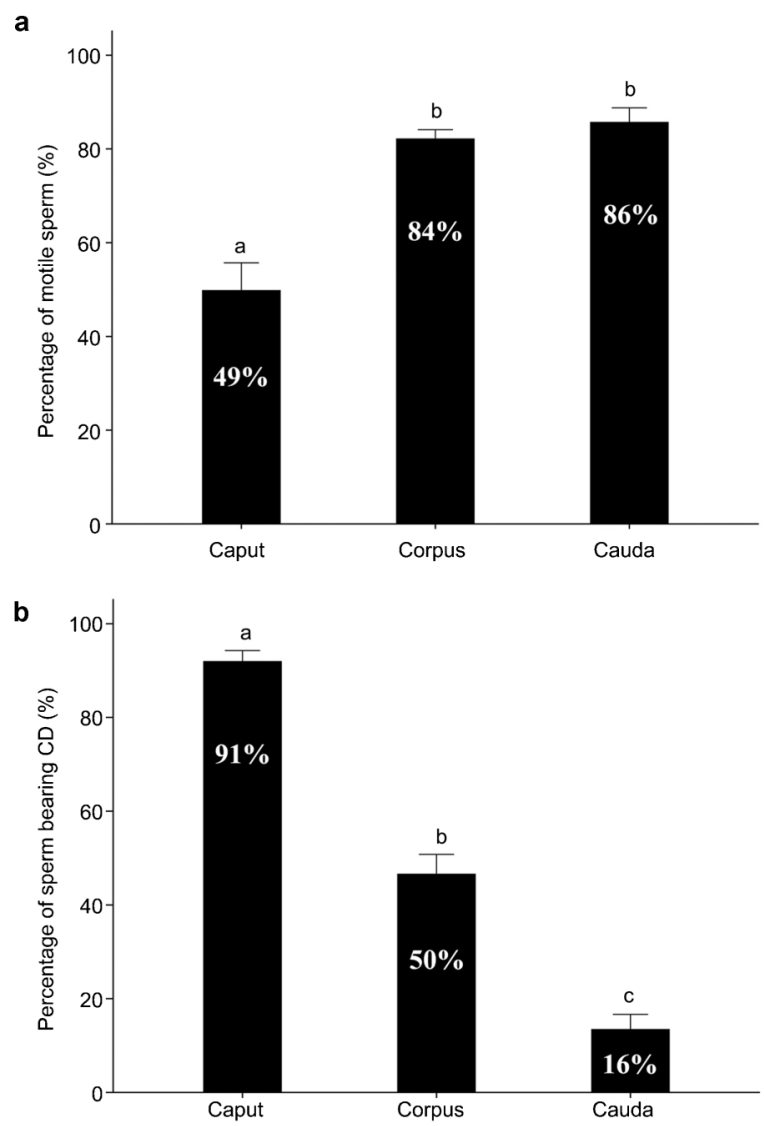

Figure 4 Motility and CD-bearing proportion of monkey epididymal spermatozoa after incubation in the HTF-HEPES at $37^{\circ} \mathrm{C}$ for 30 min. (a) Total (initial+ progressive) motility of spermatozoa collected from the caput, corpus and cauda epididymidis of monkeys. (b) Percentage of CD-bearing cells of the total motile spermatozoa collected from the caput, corpus and cauda epididymidis of monkeys. Data were collected from 10 adult male monkeys. Data are presented as mean \pm s.e.m. and datasets marked with different letters are statistically significant $(P<0.05, n=10)$. CD, cytoplasmic droplet. of time in vitro. ${ }^{21-23}$ Interestingly, we found that epididymal spermatozoa from these mutant male mice either do not have CDs or their $\mathrm{CDs}$ are located in abnormal positions (i.e., are ectopic). All three $\mathrm{KO}$ mouse lines analyzed in the present study $\left(\right.$ Spem $^{-1-}, \mathrm{Tnp}^{-/-}$and Tnp $1^{-/-} \mathrm{Hisl}^{-/-}$) display normal testicular weight, normal epididymal sperm counts, and no discernable disruptions in the seminiferous epithelium at the light microscopic levels ${ }^{21,25,26}$ (unpublished data). Spem1-null males are infertile owing to sperm deformation, characterized by sperm heads completely bent back with $100 \%$ penetrance. $^{21}$ Sperm counts and total (initial+ progressive) motility are comparable to those of wild type male mice (Figure 5a). While CDs of $61 \%-74 \%$ of wild type spermatozoa were located on the flagella (any flagellar domain), only 19\%-34\% of Spem1-null spermatozoa possessed CDs on the flagella (Figure $5 \mathbf{b}$ ). About $40 \%-53 \%$ of Spem 1-null spermatozoa had their CDs enveloping the bent heads and necks (Figure 5c), which we described previously on the basis of phase contrast microscopic observation and immunofluorescent confirmation with antibodies against proteins enriched in CDs (e.g., SPEM1, ubiquitin and 15-lipoxygenase). ${ }^{21}$ Interestingly, despite ectopic CDs the proportions of Spem1-null spermatozoa displaying initial or progressive motility were the same as those of wild type spermatozoa (Figure 5a). However, the total motility of Spem1-null spermatozoa lasted for $\sim 30 \mathrm{~min}$ in $\mathrm{HTF}$ medium at $37^{\circ} \mathrm{C}$, and within $1 \mathrm{~h}$ all spermatozoa become immotile (data not shown). These results suggest that the motility (both initial and progressive motility) may depend on CDs regardless of their location, and the development of normal and longlasting progressive motility, however, requires CDs at normal locations (i.e., mid-piece and mid-principal piece junction of the flagella) in mice.

Tnp1 KO male mice are subfertile, in part, because of reduced total sperm motility. ${ }^{25,26}$ About $65 \%$ of Tnp1-null caput epididymidal spermatozoa displayed initial motility, and this percentage was similar to that in wild type mice (Figure 5a). However, $\sim 21 \%$ Tnp1-null corpus epididymidal spermatozoa had initial motility and this proportion dropped to only $8 \%$ in the cauda epididymidis (Figure 5a), suggesting that these spermatozoa failed to maintain or develop motility competence. Tnp-1 and Hils1 double KO (Tnp1 $1^{-1-}$; Hils $\left.1^{-1-}\right)$ spermatozoa collected from the three regions of the epididymis all displayed significantly reduced initial motility compared with WT spermatozoa (Figure 5a). A significant proportion of $T n p 1^{-/-}$or $T n p 1^{-1-}$ Hisl $^{-1-}$ epididymal spermatozoa did not have CDs, and those 'CD-less' spermatozoa displayed minimal initial motility when taken from the caput epididymidis, and had completely lost their motility when taken from the corpus and cauda epididymidis, leading to 

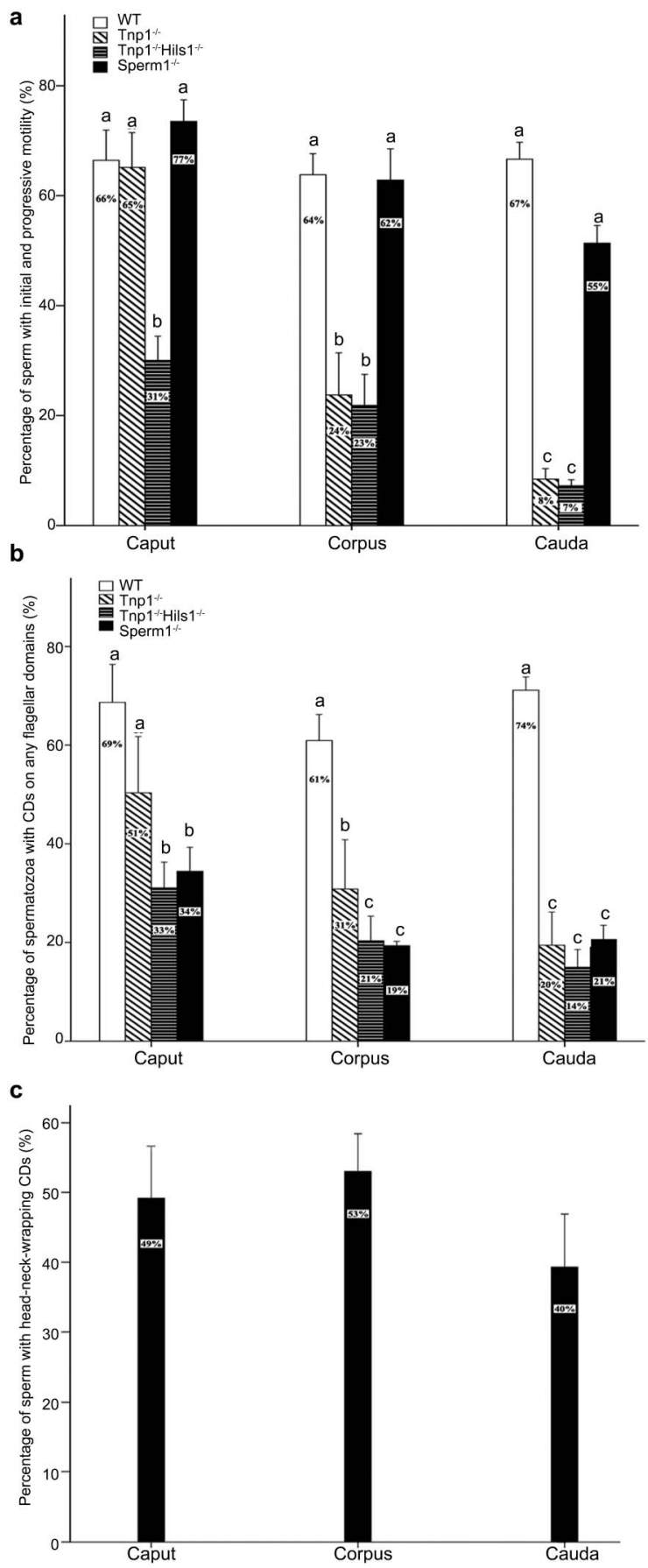

Figure 5 Motility and percentage of CD-bearing spermatozoa in WT, Tnp1 ${ }^{-/}$, Tnp $1^{-/-}-H i l s 1^{-/-}$and Spem $1^{-/-}$mice. (a) Total (initial+progressive) motility of spermatozoa collected from the caput, corpus and cauda epididymidis of WT, Tnp1 ${ }^{-/-}$, Tnp1 $1^{-1-}-H i l s 1^{-1-}$ and Spem 1 ${ }^{-1-}$ mice. (b) Percentage of spermatozoa bearing CDs on any region of the flagellum, collected from the caput, corpus and

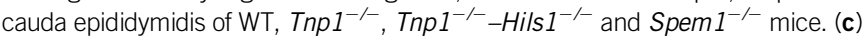
Percentage of spermatozoa with CDs wrapping around the head and the neck, collected form the Spem1 $1^{-1}$ caput, corpus and cauda epididymidis. Data are presented as mean \pm s.e.m. and datasets marked with different letters are statistically significant $(P<0.05, n=5)$. CD, cytoplasmic droplet; WT, wild-type.

significantly reduced total motility in corpus and cauda epididymidis (Figure 5b). These results suggest that defective spermiogenesis, although it may not be histologically discernable, often produces

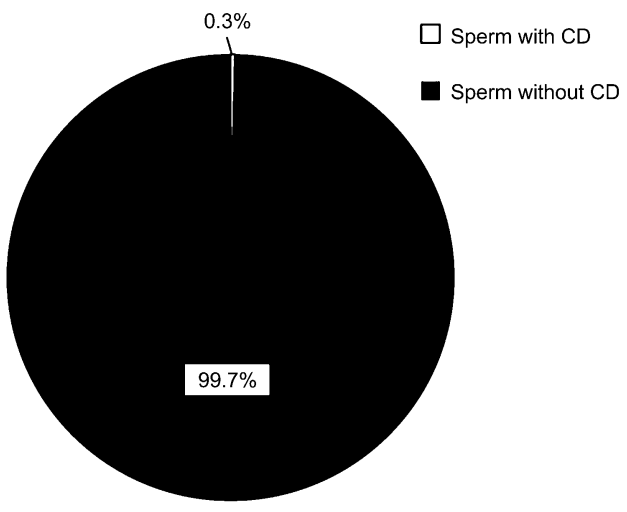

Figure 6 Ejaculated spermatozoa of C57BI/6J male mice collected from the female (CD1) reproductive tract immediately after females were mated. Data are presented as mean \pm s.e.m. and datasets marked with different letters are statistically significant $(P<0.05, n=4)$. $C D$, cytoplasmic droplet.

spermatozoa without CDs or with ectopic CDs. The absence of CDs and abnormal $\mathrm{CD}$ positions may serve as a hallmark indicative of defective spermatogenesis in the absence of histologically discernible disruptions.

\section{CDs are shed during ejaculation in mice}

In mice, almost all of the spermatozoa (99.7\%) collected from uterus minutes post-coitus contained no CDs on their flagella (Figure 6 and Supplementary Movie 4). Considering that $75 \%-88 \%$ cauda epididymidal spermatozoa still had their CDs attached to the flagella (Figure 3d), this observation suggests that CDs are mostly shed during ejaculation in mice.

\section{DISCUSSION}

CDs were first described as a bulge of cytoplasm that remains attached to the neck region of spermatozoa after spermiation. ${ }^{7}$ Subsequent ultrastructral and biochemical studies have suggested that CDs are derived from the Golgi apparatus and endoplasmic reticulum, and possess enzymatic activities. ${ }^{2-6,9,27}$ In the past two decades, studies on gene $\mathrm{KO}$ mice have suggested that $\mathrm{CDs}$ have a role in volume regulation, through which spermatozoa can adapt rapidly to osmolar challenges during their journey through the epididymis and later the female reproductive tract. ${ }^{10-12,15}$ Confusion remains in the literature ${ }^{11,17,28}$ regarding whether the presence of CDs is correlated positively or negatively with sperm function, whether CDs represent a truly functional apparatus or merely redundant cytoplasmic remnant, and whether CDs are common in all mammalian species. The controversy, in part, arises from the fact that in animal studies, epididymal spermatozoa have nearly always been used for analyzing CDs, whereas in humans, ejaculated spermatozoa are used for obvious reasons (the impracticality of collecting human epididymal spermatozoa). To clarify this confusion, we embarked on analyzing CDs on the basis of their sequential physiological destinations (caput $\rightarrow$ corpus $\rightarrow$ cauda epididymis $\rightarrow$ cervix and uterus) in mice. Our results have demonstrated that CDs represent a normal organelle present on the majority of spermatozoa in the caput epididymidis ( $90 \%$ in mice and $85 \%$ in monkeys). While spermatozoa pass through the epididymis (caput $\rightarrow$ corpus $\rightarrow$ cauda), CDs appear to migrate from an upper (neck or mid-piece) to a more distal (mid-principal junction, principal and end pieces) position in both mice and monkeys (Figure 7). More interestingly, the presence of CDs is correlated with the potential of motility development. The majority of spermatozoa displaying initial and 


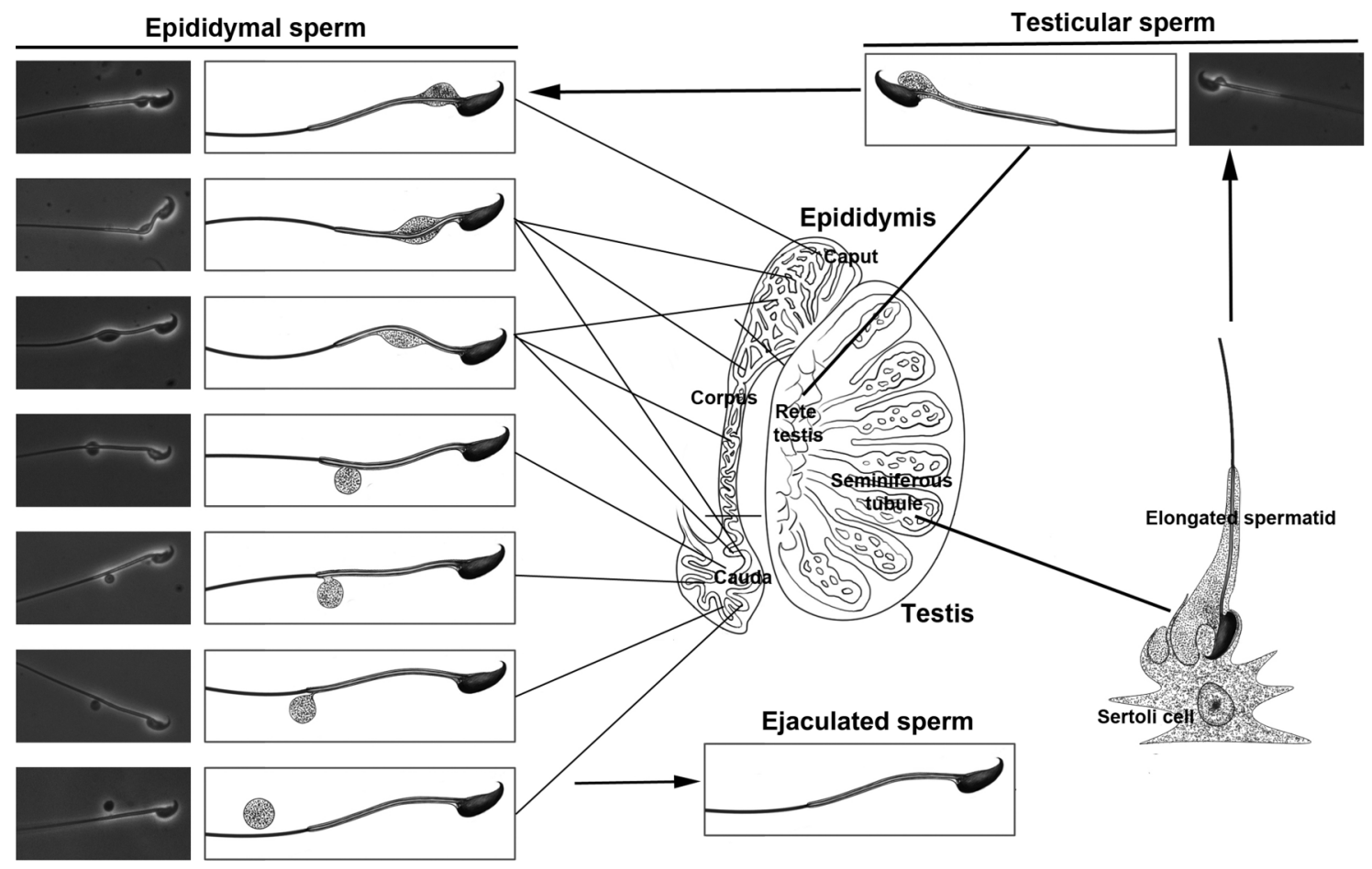

Figure 7 Schematic illustration showing positional changes of CDs on testicular, epididymal and ejaculated spermatozoa in mice. CD, cytoplasmic droplet.

progressive motility have CDs, whereas 'CD-less' spermatozoa rarely develop or maintain motility. Therefore, CDs appear to reflect sperm motility and may have a role in sperm motility development during the epididymal maturation.

The distal movement of CDs along sperm flagella is of interest and its physiological significance is, however, unclear. The fact that in mice ejaculated spermatozoa do not have CDs, whereas most of the caudal epididymidal spermatozoa possess CDs, suggests that CDs are shed during ejaculation. In monkeys, a significant proportion $(\sim 75 \%)$ of cauda epididymidal spermatozoa have already lost their CDs, suggesting that CD shedding occurs more proximally in the epididymis in monkeys than in mice. This discrepancy may reflect species differences. Nevertheless, the trend that spermatozoa start to lose their CDs when they reach the cauda epididymidis and have completely shed their CDs after ejaculation appears to be conserved between these two species (Figure 7).

Spermatozoa with typical CDs are mostly motile, e.g., the caput epididymidal spermatozoa usually display initial motility, characterized by non-progressive flagellation with symmetrical flagellum beating in $\mathrm{Ca}^{2+}$-containing phosphate-buffered solution or HTF medium, and cauda epididymidal spermatozoa develop progressive motility, characterized by vigorous flagellar waveforms that propel the cells forward. However, spermatozoa without CDs were immotile regardless where they were collected and how long they were incubated in HTF. This suggests that either these cells may have plasma membrane damage, due to cytoplasmic shedding during spermiation, or that the CDs may contain some factors that are essential for motility development. Although earlier biochemical analyses have suggested that CDs are enriched with enzymes involved in various metabolic pathways, ${ }^{2,4,27}$ systematic proteomic analyses are essential to define the proteome of CDs.

We have generated several lines of $\mathrm{KO}$ mice with late spermiogenic defects with no histologically discernable disruptions of the seminiferous epithelium. ${ }^{23}$ We have long noticed that epididymal spermatozoa in those $\mathrm{KO}$ male mice either do not bear CDs or have CDs that are not located in the common positions (i.e., middle piece or midprincipal piece junction) on the flagella. ${ }^{21,22,25,26}$ We analyzed three such $\mathrm{KO}$ mouse lines in this study, and our results support the notion that a lack of CDs or ectopic CDs appears to be indicative of spermiogenic defects in the absence of seminiferous epithelial disruption at histological level. Spem1-null spermatozoa display 'head-bent-back' phenotype with $100 \%$ penetrance. ${ }^{21}$ Electron microscopic analyses suggest that cytoplasmic removal may have been delayed in the absence of SPEM1 in elongated spermatids, leading to CD-like structures enveloping the bent head and neck in Spem1-null spermatozoa. ${ }^{21,29,30}$ The existence of CDs was further confirmed by immunofluorescence staining of proteins known to be highly abundant in CDs, including SPEM1, ubiquitin and 15-lipoxygenase. ${ }^{21}$ Spem1-null cauda epididymidal spermatozoa display initial motility and weak progressive motility, neither of which is long lasting $(<1 \mathrm{~h})$. This is consistent with our observation that as long as the CD is present on them, spermatozoa will become motile when collected into HTF, but they will lose their motility much more quickly when CDs are located in atypical positions (e.g., on the sperm head or on the head wrapping around the neck). The majority of Tnp1-null and Tnp1-Hils1 double KO spermatozoa are mostly ' $\mathrm{CD}$-free'. It is, therefore, not surprising that spermatozoa from both $\mathrm{KO}$ lines display drastically reduced total motility. Tnp1 encodes transition protein 1 (TNP1) and Hisll encodes a histone variant specifically expressed in elongated spermatids (HILS1), and both are involved in stepwise nuclear condensation and chromatin packaging processes. ${ }^{31-33}$ Tnp1 KO male mice are subfertile, ${ }^{25,26}$ and Tnp1-Hils1 double KO male mice are infertile on a mixed C57Bl/6-129Sv/Ev background (our unpublished data). Again, our data suggest that a lack of CDs is correlated with aberrant spermiogenesis. This phenomenon may be universal and further examination of CDs in other KO mouse lines with late spermiogenic disruptions may strengthen this claim. 
Although late spermiogenesis does not appear to have a checkpoint mechanism through which defective cells can be eliminated via apoptosis or other mechanisms, ${ }^{23}$ a lack of CD formation may represent a novel form of checkpoint because spermatozoa lacking them will not develop motility and thus be excluded from fertilization. However, it remains unclear how aberrant spermiogenesis leads to the lack of CD formation.

In summary, the data presented here suggest that the CD is a normal organelle exclusively present on epididymal spermatozoa in mice and monkeys, and normal CD morphology, location and migration patterns are correlated with motility development potential during epididymal sperm maturation. Abnormal CD formation, e.g., a complete lack of CDs or ectopic CDs, may be one marker indicative of defective spermiogenesis. Future proteomic and functional analyses may shed light on the true physiological role of this mysterious organelle. If the CD is essential for sperm motility development, then this structure may represent an ideal drug target for developing non-hormonal male contraceptives.

\section{AUTHOR CONTRIBUTIONS}

WY conceived and designed the study. HX, SQY and ZHZ performed the experiments and analyzed the data. SQY and WY wrote the paper.

\section{COMPETING FINANCIAL INTERESTS}

All authors declared no competing financial interests.

\section{ACKNOWLEDGMENTS}

The authors would like to thank Dr Mei-Ying Zhang and Mr Sheng-Lai Zhou in China Medical University for technical assistance, Mr R. Keegan Idler, MSc, for language editing. This work was supported in part by startup funds from the University of Nevada, Reno (to WY).

Supplementary Information accompanies the paper on Asian Journal of Andrology website (http://www.nature.com/aja).

1 Hermo L, Pelletier RM, Cyr DG, Smith CE. Surfing the wave, cycle, life history, and genes/proteins expressed by testicular germ cells. Part 1: background to spermatogenesis, spermatogonia, and spermatocytes. Microsc Res Tech 2010; 73 . 241-78.

2 Dott HM, Dingle JT. Distribution of Iysosomal enzymes in the spermatozoa and cytoplasmic droplets of bull and ram. Exp Cell Res 1968; 52: 523-40.

3 Bavdek S, Glover TD. Alkaline phosphatase in the cytoplasmic droplet of rabbit spermatozoa. J Reprod Fertil 1970; 22: 371-3.

4 Garbers DL, Wakabayashi T, Reed PW. Enzyme profile of the cytoplasmic droplet from bovine epididymal spermatozoa. Biol Reprod 1970; 3: 327-37.

5 Moniem KA, Glover TD. Alkaline phosphatase in the cytoplasmic droplet of mammalian spermatozoa. J Reprod Fertil 1972; 29: 65-9.

6 Roberts ML, Scouten WH, Nyquist SE. Isolation and characterization of the cytoplasmic droplet in the rat. Biol Reprod 1976; 14: 421-4.

7 Retzius G. Die Spermien der Huftiere. Biol Untersuch NFH 1909; 14: 163-78.

8 Burgos MH, Fawcett DW. Studies on the fine structure of the mammalian testis. I. Differentiation of the spermatids in the cat (Felis domestica). J Biophys Biochem Cytol 1955; 1: 287-300.

9 Oko R, Hermo L, Chan PT, Fazel A, Bergeron JJ. The cytoplasmic droplet of rat epididymal spermatozoa contains saccular elements with Golgi characteristics. J Cell Biol 1993; 123: 809-21.
10 Chen Q, Peng H, Lei L, Zhang Y, Kuang H et al. Aquaporin3 is a sperm water channel essential for postcopulatory sperm osmoadaptation and migration. Cell Res $2011 ; 21$ : 922-33.

11 Cooper TG. The epididymis, cytoplasmic droplets and male fertility. Asian J Androl 2011; 13: 130-8.

12 Fetic S, Yeung CH, Sonntag B, Nieschlag E, Cooper TG. Relationship of cytoplasmic droplets to motility, migration in mucus, and volume regulation of human spermatozoa. J Androl 2006; 27: 294-301.

13 Yeung $\mathrm{CH}$, Callies C, Rojek A, Nielsen S, Cooper TG. Aquaporin isoforms involved in physiological volume regulation of murine spermatozoa. Biol Reprod 2009; 80: 3507.

14 Cooper TG. Cytoplasmic droplets: the good, the bad or just confusing? Hum Reprod 2005; 20: 9-11.

15 Cooper TG, Yeung $\mathrm{CH}$. Acquisition of volume regulatory response of sperm upon maturation in the epididymis and the role of the cytoplasmic droplet. Microsc Res Tech 2003; 61: 28-38.

16 Cooper TG, Yeung CH, Fetic S, Sobhani A, Nieschlag E. Cytoplasmic droplets are normal structures of human sperm but are not well preserved by routine procedures for assessing sperm morphology. Hum Reprod 2004; 19: 2283-8.

17 Hermo L, Pelletier RM, Cyr DG, Smith CE. Surfing the wave, cycle, life history, and genes/proteins expressed by testicular germ cells. Part 3: developmental changes in spermatid flagellum and cytoplasmic droplet and interaction of sperm with the zona pellucida and egg plasma membrane. Microsc Res Tech 2010; 73: 320-63.

18 Zini A, Defreitas G, Freeman M, Hechter S, Jarvi K. Varicocele is associated with abnormal retention of cytoplasmic droplets by human spermatozoa. Fertil Steril 2000; 74: 461-4.

19 Turner RM. Moving to the beat: a review of mammalian sperm motility regulation. Reprod Fertil Dev 2006; 18: 25-38.

20 Ola B, Afnan M, Papaioannou S, Sharif K, Bjorndahl L et al. Accuracy of spermcervical mucus penetration tests in evaluating sperm motility in semen: a systematic quantitative review. Hum Reprod 2003; 18: 1037-46.

21 Zheng H, Stratton CJ, Morozumi K, Jin J, Yanagimachi R et al. Lack of Spem 1 causes aberrant cytoplasm removal, sperm deformation, and male infertility. Proc Natl Acad Sci USA 2007; 104: 6852-7.

22 Jin J, Jin N, Zheng H, Ro S, Tafolla D et al. Catsper3 and Catsper4 are essential for sperm hyperactivated motility and male fertility in the mouse. Biol Reprod 2007; 77 . 37-44.

23 Yan W. Male infertility caused by spermiogenic defects: lessons from gene knockouts. Mol Cell Endocrinol 2009; 306: 24-32.

24 Hermo L, Dworkin J, Oko R. Role of epithelial clear cells of the rat epididymis in the disposal of the contents of cytoplasmic droplets detached from spermatozoa. Am J Anatomy 1988; 183: 107-24.

25 Shirley CR, Hayashi S, Mounsey S, Yanagimachi R, Meistrich ML. Abnormalities and reduced reproductive potential of sperm from Tnp1- and Tnp2-null double mutant mice. Biol Reprod 2004; 71: 1220-9.

26 Zhao M, Shirley CR, Mounsey S, Meistrich ML. Nucleoprotein transitions during spermiogenesis in mice with transition nuclear protein Tnp1 and Tnp2 mutations. Biol Reprod 2004; 71: 1016-25.

27 Harrison RA, White IG. Glycolytic enzymes in the spermatozoa and cytoplasmic droplets of bull, boar and ram, and their leakage afte shock. J Reprod Fertil 1972, 30: $105-15$.

28 Rengan AK, Agarwal A, van der Linde M, du Plessis SS. An investigation of excess residual cytoplasm in human spermatozoa and its distinction from the cytoplasmic droplet. Reprod Biol Endocrinol 2012; 10: 92.

29 Bao J, Wu Q, Song R, Jie Z, Zheng $\mathrm{H}$ et al. RANBP17 is localized to the XY body of spermatocytes and interacts with SPEM 1 on the manchette of elongating spermatids. Mol Cell Endocrinol 2011; 333: 134-42.

30 Bao J, Zhang J, Zheng H, Xu C, Yan W. UBQLN1 interacts with SPEM 1 and participates in spermiogenesis. Mol Cell Endocrinol 2010; 327: 89-97.

31 Iguchi N, Tanaka H, Yamada S, Nishimura H, Nishimune Y. Control of mouse hils 1 gene expression during spermatogenesis: identification of regulatory element by transgenic mouse. Biol Reprod 2004; 70: 1239-45.

32 Yan W, Ma L, Burns KH, Matzuk MM. HILS1 is a spermatid-specific linker histone H1 like protein implicated in chromatin remodeling during mammalian spermiogenesis. Proc Natl Acad Sci USA 2003; 100: 10546-51.

33 Jedrzejczak P, Kempisty B, Bryja A, Mostowska M, Depa-Martynow M et al. Quantitative assessment of transition proteins 1, 2 spermatid-specific linker histone $\mathrm{H} 1$-like protein transcripts in spermatozoa from normozoospermic and asthenozoospermic men. Arch Androl 2007; 53: 199-205. 